\title{
Kinetic equation for gluons at the early stage
}

\author{
Qun Wang†, Krzysztof Redlich $\ddagger$, Horst Stöcker $\dagger$ and Walter \\ Greiner $\dagger$ \\ † Institute für Theoretische Physik, J. W. Goethe-Universität, D-60054 Frankfurt, \\ Germany \\ $\ddagger$ Theory Division, CERN, CH-1211 Geneva 23, Switzerland
}

\begin{abstract}
.
We derive the kinetic equation for pure gluon QCD plasma in a general way, applying the background field method. We show that the quantum kinetic equation contains a term as in the classical case, that describes a color charge precession of partons moving in the gauge field. We emphasize that this new term is necessary for the gauge covariance of the resulting equation.
\end{abstract}

Heavy ion collisions at ultra-relativistic energies are widely expected to be a laboratory to study the formation and properties of highly excited QCD matter in the deconfined Quark-Gluon Plasma (QGP) phase [1]. The QGP is considered as a partonic system being at (or close to) local thermal equilibrium. Thus, to study the conditions for the possible formation of QGP in heavy ion collisions one needs to address the question of thermalization of the initially produced partonic medium [2]. On the theoretical level this requires the formulation of kinetic equations [3] involving color degrees of freedom and the non-Abelian structure of QCD dynamics. Different models for the initial conditions in ultra-relativistic heavy ion collisions suggest that at the early stage the medium is dominated by gluon degrees of freedom [4]; the kinetic equation for the pure gluon plasma is thus of special interest.

The kinetics of a particle moving in a non-Abelian gauge field was first studied for $\mathrm{SU}(2)$ symmetry [5]. The equation of motion derived in [5] made it clear that in the presence of the gauge field the color charge of the particle keeps rotating or undergoing precession with time. Consequently, there is an explicit term in the classical kinetic equation, which describes the time evolution of the color charge of partons. Applying a typical method to derive a semi-classical transport equation from the quantum theory, which is contained in the formulation of the equation of motion for the Wigner function and its subsequent gradient expansion [6, 7, 8], one loses the explicit presence of the color charge precession because the obtained transport equations are not most simplified. The author of Ref. [9] simplified the kinetic equation for gluons based on Ref. [8]. However the simplification is under the assumption of a specific form of the covariant Green function (actually it is a special case of this work), and the color precession term was not explictly and transparently interpreted. 
The usual treatment of the gluon transport equation is based on the decomposition of the gluon field into a mean field and a quantum fluctuation, or into a soft and a hard part. Under this approximation the gluon transport equation then describes the kinetics of the quanta in the classical mean field [6, 10]. This picture is somehow similar to the one used while studying the energy loss of a fast parton moving in the soft mean field [11, 12]. Since this decomposition is not rigorous, the explicit gauge invariance of the formalism remains unclear. To include the classical chromofield into QCD in a proper way, one uses the background field method of QCD (BG-QCD) introduced by DeWitt and 't Hooft [13, 14, 15. The advantage of BG-QCD is that it is formulated in an explicit gauge invariant manner.

One of the first attempts to derive the gluon transport equation in BG-QCD was presented in [16]. But the obtained equation is not transparent. The most recent work has been done by Blaizot and Iancu [17, 18] in the context of the close-time-path (CTP) formalism. There, however, the authors focus on formulating the transport equation in the vicinity of equilibrium.

In this work, we use the CTP method to derive the kinetic equation for the gluon plasma in BG-QCD. Our derivation is going beyond previous results as it is quite general and not limited to the vicinity to equilibrium. One of the most important features of the kinetic equation derived here is that a color charge precession term is explicitly present, similar to the one in the classical equation [5, 6, 7]. We demonstrate that this term is necessary to guarantee the gauge covariance of the resulting Vlasov equation.

In the following we use $g_{\mu \nu}=\operatorname{diag}(1,-1,-1,-1)$ as the metric tensor, and we write Lorentz indices as subscripts and color ones as superscripts for the relevant quantities. For the gauge field and its field tensor we also denote: $A_{\mu} \equiv A_{\mu}^{a} T^{a}$ and $F_{\mu \nu}[A] \equiv F_{\mu \nu}^{a}[A] T^{a}$, where $\left(T^{a}\right)^{i j}=i f^{i a j}$ are the generators of the $S U(3)_{c}$ adjoint representation. The two-point Green function (GF) or self-energy (SE) are treated as matrices, and thus the color and/or Lorentz indices are sometimes omitted.

Applying the background field method, we decompose the conventional gluon field into the sum of a classical background part $A$ and a quantum fluctuation $Q$. Including the appropriate gauge fixing and ghost term for the background gauge $D_{\mu}^{i j}[A] Q_{\mu}^{j}=0$, the BG-QCD Lagrangian reads [15

$$
\begin{aligned}
\mathcal{L}= & -\frac{1}{4} F_{\mu \nu}^{i}[A+Q] F_{\mu \nu}^{i}[A+Q]-\frac{1}{2 \alpha}\left(D_{\mu}^{i j}[A] Q_{\mu}^{j}\right)^{2} \\
& +\bar{C}^{i} D_{\mu}^{i j}[A] D_{\mu}^{j k}[A+Q] C^{k},
\end{aligned}
$$

where $D_{\mu}^{i j}[A(x)]=\left[\partial_{x \mu}-i g A_{\mu}(x)\right]^{i j}$ is the covariant derivative, $C^{i} / \bar{C}^{i}$ are the ghost/anti ghost fields and $\alpha$ is the gauge fixing parameter.

The above Lagrangian is invariant under the local gauge transformation of type I (type II transformation is irrelevant to the current problem) where the background field transforms as a conventional gauge fields, $A_{\mu}^{\prime}=U A_{\mu} U^{-1}+i g^{-1} U \partial_{\mu} U^{-1}$, while the gluon and ghost field transform as a matter field, $Q_{\mu}^{\prime}=U Q_{\mu} U^{-1}$ [15]. Here $U(x)=\exp \left[i g \omega^{a}(x) T^{a}\right]$ is the transformation matrix. 
The non-equilibrium dynamics is usually described in the CTP formalism [19]. Here the action can be written as $S_{C T P}=S_{+}-S_{-}+K\left(A_{ \pm}, Q_{ \pm}\right)$where all fields in $S_{ \pm}$are defined on the positive/negative time branches and $K\left(A_{ \pm}, Q_{ \pm}\right)$is the kernel incorporating initial state correlations. The GF for the gluon has four components: $\left[G^{++}, G^{+-}, G^{-+}, G^{--}\right] \equiv\left[G^{F}, G^{<}, G^{>}, G^{\bar{F}}\right]$, marked by the positive or the negative time, respectively. At the tree level there are only vertices with all positive or all negative time variables, which differ by a sign. The non-local kernel $K\left(A_{ \pm}, Q_{ \pm}\right)$appearing in $S_{C T P}$ is non-vanishing only at the initial time and plays the role of the initial density matrix. If the initial time is not in the remote past, it introduces a time dependence to the distribution function, and consequently the time-translational invariance is lost [20. It is interesting to note that the situation here is similar to the pinch singularity discussed in [21.

In the CTP formalism, choosing the physical representation for the GF and SE , the Dyson-Schwinger equation (DSE) for $G^{C}$ reads

$$
\begin{aligned}
\mathcal{D}\left(x_{1}\right) G^{C}\left(x_{1}, x_{2}\right)= & -\int d^{4} x^{\prime}\left[\Pi^{C}\left(x_{1}, x^{\prime}\right) G^{A}\left(x^{\prime}, x_{2}\right)\right. \\
& \left.+\Pi^{R}\left(x_{1}, x^{\prime}\right) G^{C}\left(x^{\prime}, x_{2}\right)\right], \\
G^{C}\left(x_{1}, x_{2}\right) \mathcal{D}^{\dagger}\left(x_{2}\right)= & -\int d^{4} x^{\prime}\left[G^{R}\left(x_{1}, x^{\prime}\right) \Pi^{C}\left(x^{\prime}, x_{2}\right)\right. \\
& \left.+G^{C}\left(x_{1}, x^{\prime}\right) \Pi^{A}\left(x^{\prime}, x_{2}\right)\right],
\end{aligned}
$$

where the differential operators $\mathcal{D}$ and $\mathcal{D}^{\dagger}$ in the Feynman gauge $\S(\alpha=1)$ are expressed by:

$$
\begin{aligned}
& \mathcal{D}_{\rho \sigma}^{h i}=g_{\rho \sigma} D_{\mu}^{h a}[A] D_{\mu}^{a i}[A]+2 g f^{h a i} F_{\rho \sigma}^{a}[A], \\
& \mathcal{D}_{\rho \sigma}^{\dagger ; h i}=g_{\rho \sigma} D_{\mu}^{\dagger ; h a}[A] D_{\mu}^{\dagger ; a i}[A]+2 g f^{h a i} F_{\rho \sigma}^{a}[A],
\end{aligned}
$$

where $D_{\mu}^{\dagger ; i j}[A(x)] \equiv\left[\overleftarrow{\partial}_{x \mu}+i g A_{\mu}(x)\right]^{i j}$ is the conjugate covariant derivative where the differential operator acts on the function on its left.

In the evolution of the gluon field one distinguishes different scales, which characterize quantum and soft collective motion. We introduce a mass parameter, $\mu$, as the separation point of the quantum and the kinetic scale. In the weak coupling limit $g \ll 1$, the scale of collectivity $\sim 1 /(g \mu)$ is much larger than the typical extension of hard quantum fluctuations $\sim 1 / \mu$. The effect of the classical field $A$ on the hard quanta involves the coupling $g A$ to the hard propagator and is of the size of the soft wavelength $\sim 1 /(g \mu)$. The above separation of scales is the basis for the gradient expansion where one expresses all 2-point GF's in terms of the relative $y$ and the central $X$ coordinate. Here are some typical scales: $y=x_{1}-x_{2} \sim 1 / \mu, X=\left(x_{1}+x_{2}\right) / 2 \sim 1 /(g \mu), A(X) \sim \mu$ and $F[A(X)] \sim g \mu^{2}$.

$\ddagger$ In the physical representation the elements of GF and SE matrices are expressed by the symmetric $(\mathrm{C})$, the retarded $(\mathrm{R})$ and the advanced $(\mathrm{A})$ components. They are related to the original components by the following transformations: $G^{A}=G^{F}-G^{>}, G^{R}=G^{F}-G^{<}, G^{C}=G^{>}+G^{<}$(the same relations hold for the SE $\Pi^{C / R / A}$ up to a sign in front of $\Pi^{>}$and $\left.\Pi^{<}\right)$.

$\S$ In fact due to gauge conditions: $D_{\mu}^{i j}\left[A\left(x_{1}\right)\right] G_{\mu \nu}^{j k}\left(x_{1}, x_{2}\right)=0$ and $G_{\mu \nu}^{i j}\left(x_{1}, x_{2}\right) D_{\nu}^{\dagger ; j k}\left[A\left(x_{2}\right)\right]=0$, Eqs. (2.3) are independent of the gauge parameter $\alpha$. 
In order to obtain the gauge-covariant kinetic equation one uses the gauge-covariant Wigner function $\tilde{G}(X, y)\left(\tilde{G}^{C}, \tilde{G}^{>}\right.$or $\left.\tilde{G}^{<}\right)$defined by

$$
G\left(x_{1}, x_{2}\right)=V\left(x_{1}, X\right) \tilde{G}(X, y) V\left(X, x_{2}\right),
$$

where $V\left(z_{1}, z_{2}\right)=T_{P} \exp \left(i g \int_{P ; z_{2}}^{z_{1}} d z_{\mu} A_{\mu}\right)$ denotes a Wilson link with respect to the classical background field. One can also define the Wilson link as a functional of $A+Q$, but it is a much more complicated case and beyond the scope and subject of this work.

The covariant Wigner function $\tilde{G}(X, y)$ transforms as $U(X) \tilde{G}(X, y) U^{-1}(X)$; only the transformation at a single point $X$ is thus relevant. For $G\left(x_{1}, x_{2}\right)$, however, the gauge transformation involves two points and therefore is not gauge-covariant.

Taking the difference between Eq.(22) and Eq.(3), we derive the following kinetic equation in terms of the gauge covariant Wigner function $\tilde{G}^{C}(X, y)$ :

$$
\begin{aligned}
& q \cdot \partial_{X} \tilde{G}_{\alpha \gamma}^{C}+i g\left(\tilde{G}_{\alpha \gamma}^{C} q \cdot A-q \cdot A \tilde{G}_{\alpha \gamma}^{C}\right) \\
& +\frac{1}{2} g q_{\nu} F_{\nu \lambda}\left(\partial_{q \lambda} \tilde{G}_{\alpha \gamma}^{C}\right)+\frac{1}{2} g\left(\partial_{q \lambda} \tilde{G}_{\alpha \gamma}^{C}\right) q_{\nu} F_{\nu \lambda} \\
& +g\left(F_{\alpha \beta} \tilde{G}_{\beta \gamma}^{C}-\tilde{G}_{\alpha \beta}^{C} F_{\beta \gamma}\right)=0,
\end{aligned}
$$

where $\tilde{G}_{\alpha \gamma}^{C} \equiv \tilde{G}_{\alpha \gamma}^{C}(X, q)$ is the Fourier transform of $\tilde{G}(X, y)$ with respect to $y$ : $\tilde{G}(X, q)=$ $\int d^{4} y \tilde{G}(X, y) e^{i q y}$.

The above equation is located at the collective coordinate $X$ and is gauge-covariant under the local gauge transformation $U(X)$, i.e. it transforms as $U(\cdots) U^{-1}$. Indeed, noting that both $F_{\mu \nu}$ and $\tilde{G}_{\alpha \gamma}^{C}$ are gauge-covariant and $\partial_{q \mu}$ does not affect $U$, it is obvious that the last three terms are gauge-covariant. With some algebra, we can also prove the first two terms preserve gauge covariance too.

The quantum kinetic equation Eq.(7) is derived in a quite general manner without requirements or assumptions that the QCD plasma appears close to equilibrium. In the following we show that Eq.(7) is a natural quantum generalization of the classical Vlasov equation. In particular the color charge precession will be explicitly identified in the quantum description of color charge kinetics given by Eq.(7).

The classical kinetic equation for the color singlet distribution function $f(x, p, Q)$ has the following form [5, 6, 7]:

$$
\begin{aligned}
& p_{\mu}\left[\partial_{\mu}-g Q^{a} F_{\mu \nu}^{a} \partial_{p \nu}\right. \\
& \left.-g f^{a b c} A_{\mu}^{b}(x) Q^{c} \partial_{Q^{a}}\right] f(x, p, Q)=0,
\end{aligned}
$$

where $Q^{a}$ is the classical color charge and $a=1, . ., N_{c}^{2}-1$.

Comparing Eq.(8) with the quantum expression Eq.(7) it is clear that the color singlet distribution function $f$ is replaced by the gauge-covariant Wigner function $\tilde{G}^{C}$ which is a color matrix in the adjoint representation. One can also recognize that the first, third and fourth terms of Eq.(7) are the quantum generalization of the first two terms in Eq.(8). The last term in Eq.(7) appears from the covariant operators \ and hence is not present in the classical equation.

\| This term can be written in different form by using generators of the Lorentz transformation in vector representation. Similar term can be found for the quark, but expressed through generators in spinor representation. 
Particularly interesting is the appearance of the second term in Eq.(7). We have seen that its presence is crucial to assure the gauge covariance of the Vlasov equation. In addition this term has an interesting physical meaning. It is the quantum analogue to the color charge precession in the classical kinetic equation. To see this more clearly, one can expand $\tilde{G}_{\alpha \beta}^{C}(X, q)$ with respect to the generators $T^{a}$ of the adjoint representation as follows

$$
\begin{aligned}
\tilde{G}_{\alpha \beta}^{C}(X, q)= & N_{\alpha \beta}(X, q)+T^{a} N_{\alpha \beta}^{a}(X, q) \\
& +T^{a} T^{b} N_{\alpha \beta}^{a b}(X, q)+\cdots,
\end{aligned}
$$

where $T^{a}$ are quantum analogues to the classical color charges $Q^{a} ; N_{\alpha \beta}(X, q)$ is the color singlet and $N_{\alpha \beta}^{a}(X, q)$ the color octet function, etc. This expansion is similar to the multipole expansion of an electromagnetic source in electrodynamics. In the lowest order approximation, one can keep only the first two terms in Eq.(9) and neglect all others. Then the second term of Eq.(17) becomes

$$
i g\left(\tilde{G}_{\alpha \gamma}^{C} q \cdot A-q \cdot A \tilde{G}_{\alpha \gamma}^{C}\right) \simeq-g f^{a b c} q_{\mu} A_{\mu}^{b} T^{c} \partial_{T^{a}} \tilde{G}_{\alpha \gamma}^{C}
$$

which is just the classical color precession term in Eq.(8).

Since we know that $D_{X} \sim g A(X) \sim g \mu$, the first two terms of Eq.(7), i.e. $q \cdot \partial_{X} \tilde{G}_{\alpha \gamma}^{C}$ and the color precession term, are at leading order $O\left(g \mu^{2}\right)$, while other terms are at subleading order $O\left(g^{2} \mu^{2}\right)$. In the vicinity of equilibrium the natural scale in the system is the temperature $T$. The mean distance between particles is of the order of $\sim 1 / T$, while $1 /(g T)$ characterizes the scale of collective excitations [17, 18]. For small coupling constant $g$ these two scales are well separated. The covariant Wigner functions can be expanded around their equilibrium values: $\tilde{G}=\tilde{G}^{(0)}+\delta \tilde{G}$, where the equilibrium function $\tilde{G}^{(0)}$ is a color singlet and the fluctuation $\delta \tilde{G} \sim g^{2} \tilde{G}$. Typical scales are $q \sim T$, $D_{X} \sim g^{2} T, g F \sim\left(D_{X}\right)^{2} \sim g^{4} T^{2}$. Thus, at leading order, only the first term of Eq.(17) survives and the precession term vanishes due to the color-singlet nature of $\tilde{G}^{(0)}$.

In summary, by applying the closed-time-path formalism we have derived the gluon kinetic equation in the background gauge of QCD. Equation (17) is quite general and is not limited to the vicinity of equilibrium. It is a kinetic equation with respect to a gauge-covariant Wigner function, which is a matrix in adjoint color space. Therefore it contains many non-Abelian features which are absent from the well known classical equation. A notable feature is that, as in the classical case, it contains a term that corresponds to the color precession, the non-Abelian analogue to the Larmor precession for particles with magnetic moments in a magnetic field. This term was not explicitly shown before in the formulation of a Boltzmann equation for the QCD plasma. We find that this term is necessary to the gauge covariance of the kinetic equation.

\section{Acknowledgments}

One of us Q.W. acknowledges financial support of the Alexander von HumboldtFoundation $(\mathrm{AvH})$ and appreciated help from D. Rischke. This work is partially funded by DFG, BMBF and GSI. K.R. acknowledges a partial support of the Polish Committee 
for Scientific Research (KBN-2P03B 03018). Stimulating comments and discussions with R. Baier, J.-P. Blaizot, E. Iancu and S. Leupold are acknowledged. Our special thanks go to X.-N. Wang for his help and interest in this work.

\section{References}

[1] For a recent review, see eg. S.A. Bass, M. Gyulassy, H. Stocker and W. Greiner; J. Phys. G25, R1 (1999); H. Satz, Rep. Prog. Phys. 63, 1511 (2000).

[2] R. Baier, A.H. Mueller, D. Schiff and D.T. Son, Phys. Lett. B502, 51 (2001).

[3] For a recent review, see eg. J.-P. Blaizot and E. Iancu, hep-ph/0101103, Phys. Rept., to appear.

[4] L. McLerran and R. Venugopalan, Phys. Rev. D49, 2233 (1994); D49, 3352 (1994); D50, 2225 (1994); K.J. Eskola, K. Kajantie, P.V. Ruuskanen and K. Tuominen, Nucl. Phys. B570, 379 (2000).

[5] S. K. Wong, Nuovo Cimento A65, 689 (1970).

[6] H.-Th. Elze and U. Heinz, Phys. Rep. 183, 81 (1989); U. Heinz, Ann. Phys. 161, 48 (1985); 168, 148 (1986).

[7] H.-Th. Elze, M. Gyulassy and D. Vasak, Nucl. Phys. B276, 706 (1986).

[8] H.-Th. Elze, Z. Phys. C38, 211 (1988).

[9] S. Mrowczynski, Phys. Rev. D39, 1940 (1989).

[10] K. Geiger, Phys. Rev. D56, 2665 (1997).

[11] M. Gyulassy and X.-N. Wang, Nucl. Phys. B420, 583 (1994); M. Gyulassy, X.-N. Wang and M. Plümer, Phys. Rev. D51, 3436 (1995); X.-F. Guo, and X.-N. Wang, Phys. Rev. Lett. 85, 3591 (2000).

[12] R. Baier et al., Nucl. Phys. B483, 291 (1997); B484, 265 (1997); R. Baier et al., Nucl. Phys. B531, 403 (1997).

[13] B.S. DeWitt, Phys. Rev. D162, 1195 and 1239 (1967).

[14] G. 't Hooft, Nucl. Phys. B62, 444 (1973).

[15] L.F. Abbott, Nucl. Phys. B185, 189 (1981); R.B. Sohn, Nucl. Phys. B273, 468 (1986); H. KlubergStern and J.B. Zuber, Phys. Rev. D12, 482 (1975).

[16] H.-Th. Elze, Z. Phys. C47, 647 (1990).

[17] J.-P. Blaizot, and E. Iancu, Nucl. Phys. B557, 183 (1999).

[18] J.-P. Blaizot and E. Iancu, Nucl. Phys. B417, 608 (1994); B570, 326 (2000); Phys. Rev. Lett. 70, 3376 (1993).

[19] J. Schwinger, J. Math. Phys. 2, 407 (1961); L.V. Keldysh, Sov. Phys. JETP 20, 1018 (1965); G.Z. Zhou, Z.B. Su, B.L. Hao and L. Yu, Phys. Rep. 118, 1 (1985); E. Calzetta and B.L. Hu, Phys. Rev. D37, 2878 (1988).

[20] Q. Wang, K. Redlich, H. Stöcker and W. Greiner, in preparation.

[21] T. Altherr and D. Seibert, Phys. Lett. B333, 149 (1994); R. Baier, M. Dirks, K. Redlich and D. Schiff, Phys. Rev. D56, 2548 (1977); C. Greiner and S. Leupold, Eur. Phys. J. C8, 517 (1999); I. Dadic, Phys. Rev. D59, 125012 (1999); D63, 025011 (2001). 